\title{
Optimization Design of Lightning Protection Circuit for LED Driving Power Supply
}

\author{
CHANG Tian Hai ${ }^{1}$ a , WEI Xiao Qun ${ }^{2, b}$ \\ ${ }^{1}$ School of Electronic and Information Engineering, South China University of Technology, \\ Guangzhou 510641, China \\ ${ }^{2}$ School of Electronic and Information Engineering, South China University of Technology, \\ Guangzhou 510641, China \\ athchang@scut.edu.cn, ${ }^{b}$ xiaoqun_wei@126.com
}

\begin{abstract}
Keywords: LED; driving power supply; lightning protection circuit; surge.
Abstract. Light emitting diode( LED) is widely used because of its high efficiency, environmental protection, long life and so on. However, the use of LED is affected by the performance of the driving power supply, especially in the complex and poor working environment. Therefore, it is very important to improve the stability of the driving power supply, and to add the lightning protection circuit to the LED driver technology. According to the basic requirements of multilevel surge protection, this paper analyses the lightning protection circuit of a common LED driving power supply.
\end{abstract}

\section{Introduction}

With the rapid development of LED technology, LED has been widely used because of its high efficiency, long life and environmental protection and so on [1-3]. The use of LED also needs to match the corresponding drive power. However, the life of the drive power supply is much less than the life of the LED light source, which severely restricts the overall life of LED lamps. In addition, LED drive power supply environment is sometimes relatively poor, especially in the natural environment of lightning [4].

At present, the protection circuit almost will be put in the input of the LED driver power supply. The components that used to ensure the safe and stable operation of the circuit have more types, which including gas discharge tube, a voltage sensitive resistor, diode and positive temperature coefficient thermistor (PTC), but the precise research on lightning protection circuit model is still few. People just based on a single or a few parameters to design the circuit, failing to consider the overall performance of the circuit, insufficient element short life, lightning residual pressure is too large [5-6].

To solve the above problems, this paper puts forward to optimize the lightning protection circuit, the circuit model is built by simulation software to verify the design, to provide a more comprehensive reference to the power supply protection circuit, according to the basic requirements of multi-level protection surge, lightning protection circuit principle analysis of a common LED driver and the existing problems.

\section{Lightning Model Analysis}

Lightning is a common natural phenomenon in life, which can be divided into direct lightning, thunder and lightning conduction three [7]. One of the main hazards of LED drive power is lightning, and it can generate high frequency impulse voltage spikes in gas discharge moment, which have a great impact on the reliability of electromagnetic wave on LED drive power supply voltage caused by the impact of the direct driving power in the Central Plains breakdown components etc. And frequent discharge generated strong electromagnetic waves will bring common mode and differential mode interference, affecting the normal operation of equipment. Although the instantaneous voltage generated by lightning is very high, the main reason for the damage caused by the drive power is the 
induced current surge. In this paper, the 8/20us impact model of switching electromagnetic pulse is defined in IEC61000, as shown in Figure 1

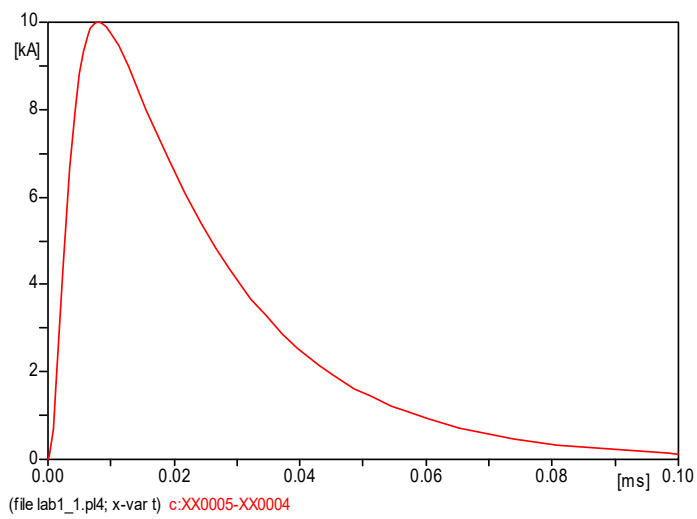

Fig. 1 8/20us surge wave

$8 / 20$ us standard lightning current waveform is a generalized surge current waveform, meaning that it is considered at the leading edge of time of about 8us, depending on the half peak time of about 20us.

\section{Lightning protection circuit design}

The common lightning protection circuit mainly adopts the voltage sensitive resistor and a gas discharge tube directly connected in series or in parallel using [8]. With the varistor and multistage gas discharge tube used to enhance performance of lightning protection circuit. Varistor and a gas discharge tube connection includes two series and parallel way. Studies showed that [9], parallel mode can not solve the problem of gas stream discharge may occur, the protection is not suitable for the AC power supply system; series, due to the larger varistor parasitic capacitance and parasitic capacitance of gas discharge tube is small, which makes the whole series branch total capacitance becomes very small, but the gas discharge tube is turned on, the voltage is low, the residual voltage branch falls on varistor, if the current is greater than the varistor gas discharge tube arc continuous current size, cannot be promptly cut off current. In addition, the varistor discharge tube in parallel multistage gas position will not affect the residual pressure value changes, only the size of the residual pressure and impulse voltage. At the same time, when the components impact times increasing, multistage gas discharge tube will first appear aging deterioration.

Aiming at the existing problems in the traditional lightning protection circuit, this paper designs the lightning protection circuit a good overall performance, the core element includes a varistor, a gas discharge tube and the positive temperature coefficient thermistor, circuit principle diagram as shown in figure 2.

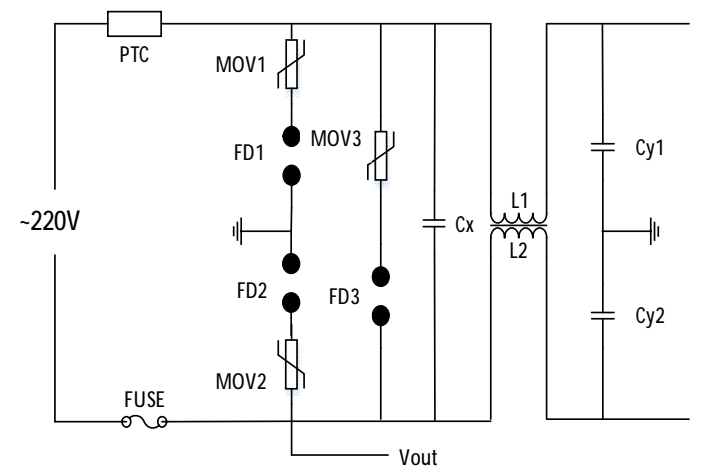

Fig.2 Optimal lightning protection circuit

Small circuit unit of the circuit including the discharge tube series three varistor and the gas, to form a composite symmetrical circuit, coupled with a positive temperature coefficient thermistor and a fuse tube, realize common mode and differential mode protection. The parallel connection of the 
pressure sensitive resistance can not only prolong the service life of the whole, but also can not cause the fire caused by the short circuit failure of the pressure sensitive resistor.

Circuit principle: MOV1 varistor and a gas discharge tube FD1 series grounding to discharge surge current L line lightning strike; MOV2 varistor and a gas discharge tube FD2 series grounding is used to discharge interference signal transmitted from the DC power supply port Vout. When the varistor fails, the corresponding series gas discharge tube branch will isolate with the rear power, will not cause the discharge of fire phenomena. PTC is mainly used to limit the current in the loop; FUSE is used to isolate the power supply when the circuit is too large.

\section{Simulation results and analysis}

In order to verify the feasibility of the designed circuit and the related performance, it is necessary to establish an accurate model of the components used in the circuit, and then use the software to simulate. At present, the commonly used simulation tools are power system electromagnetic transient analysis software EMTP. The IEEE working group gives the equivalent model of the [10], as shown in figure 3.

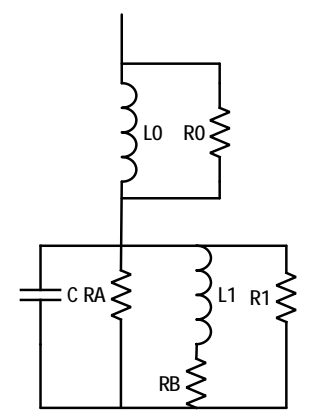

Fig.3 Equivalent model of varistor

Change the model of RA and RB, according to the circuit of Figure 2 simulation, the simulation data obtained as shown in table 1.

Table 1 Comparison between simulation data and calculation data

\begin{tabular}{|c|c|c|c|c|c|c|}
\hline \multicolumn{3}{|c|}{ L-GND } & \multicolumn{2}{|l|}{ N-GND } & \multicolumn{2}{|l|}{ L-N } \\
\hline Item & Current(k & Residual & Current(kA & Residual & Current(kA & Residual \\
\hline IIEIII & A) & voltage $(\mathrm{V})$ & ) & voltage $(\mathrm{V})$ & ) & voltage $(\mathrm{V})$ \\
\hline Calculation & 3.00 & 236 & 3.00 & 884 & 3.00 & 457 \\
\hline Simulation & 3.33 & 264 & 3.27 & 928 & 3.20 & 478 \\
\hline
\end{tabular}

The table shows that the data and calculated data of small errors in the $8 / 20 \mathrm{us}$ simulation, $3 \mathrm{kA}$ impulse current residual voltage of the lightning protection circuit is less than $1000 \mathrm{~V}$, meet the requirements of the standard, can effectively protect the stability of driving circuit.

All manuscripts must be in English, also the table and figure texts, otherwise we cannot publish your paper.

\section{Conclusions}

According to the basic requirements of surge protection multi-stage lightning protection circuit, this paper analyzed the principle of a LED drive power and the existing problems, put forward to optimize the lightning protection circuit, the circuit simulation software to verify the model.

\section{Acknowledgements}

This work was financially supported by the China Academy of Space Technology Development Project (x2dxD8140090). 


\section{References}

[1] Xu Sheng, Xu Yuzhen, Chen Enguo, et al. Study on the model of high power LED volt ampere characteristics [J]. Photoelectron and Laser, 2015 (11): 2076-2082.

[2] Lay-Ekuakille A, Vergallo P, Morello R, et al. Indoor air pollution system based on LED technology[J]. Measurement, 2014, 47:749-755.

[3] Han H V, Lin H Y, Lin C C, et al. Resonant-enhanced full-color emission of quantum-dot-based micro LED display technology.[J]. Optics Express, 2015, 23(25).

[4] Su Ping. The lightning wave intrusion of low voltage power supply lightning protection [J]. Guangxi meteorology, 2002, 23 (3): 56-57.

[5] Zhongshan Zhou, Zhang Yunfeng, Chen Puyang,. Multilevel surge protector on the load test effectiveness analysis [J]. Insulators and surge arresters, 2014 (4): 49-54.

[6] Li Xiangchao, Chen Puyang, Xule,. Multi electrode gas discharge tube and $\mathrm{ZnO}$ varistor properties [J]. Insulators and surge arresters matching 2015 (3) employment sectors Journal of: 48-56.

[7] Zhang Yijun, Zhou Xiuji. Review and development of lightning research [J]. Journal of Applied Meteorology, 2006, 17 (6): 829-834.

[8] Hu Jun, long and long, He Jinliang, et al. Analysis of the influence factor of the residual voltage ratio of the $\mathrm{ZnO}$ based on the [J]. high voltage technology, 2011, 37 (3): 555-561.

[9] He Jinliang, Liu Jun, Hu Jun, et al. Research progress of $\mathrm{ZnO}$ based on for the surge arrester in power system [J]. high voltage technology, 2011, 37 (3): 634-643.

[10] Tominaga S, Azumi K, Shibuya Y, et al. Protective Performance of Metal Oxide Surge Arrester Based on the Dynamic V-I Characteris-tics[J]. IEEE Transactions on Power Apparatus \& Systems, 1979, PAS-98(6):1860-1871. 